Financiar la igualdad, ina obligacion implicita del Estado PARA ATENDER A LAS SITIACIONES DE DEPENDENCLA

Josep Fargas Fernández*

RESUMEN:

La Ley de promoción de la autonomia personal y de atención a las personas en situación de dependencia. sigue las tendencias internacionales actuales en pro de la extensión de la Seguridad Social; entendida ésta como un sistema amplio e integrador de diversos subsistemas -entre ellos el nuevo SAAD-, que proporciona prestaciones económicas y servicios personales o sociales, y promueve la coordinación de todos los poderes puíblicos. A través de esta Ley básica, el Estado garantiza a los ciudadanos un nivel de protección minimo, comin e igual. al que tiene la obligación implicita (es la otra cara de la teoría de los poderes implicitos) de aportar los recursos financieros necesarios para que tal igual protección sea real y efectiva.

\title{
Paitabras Clave:
}

Dependencia. Servicios Sociales. Seguridad Social. Poderes implicitos. Obligaciones implicitas. Competencias constitucionales. Responsabilidad del Estado. Comunidades Autónomas.

- Profesor Titular de Derecho del Trabajo y S.S. Universidad Pompeu Fabra- Barcelona • josep.fargas@upf.edu 
ABSTRACT:

The Law of promotion of the personal autonomy and attention to the people in a dependancy situation, follows the international tendencies to the extension of the Social Security: understood this one like an ample and integrating system of diverse subsystems - among them the new SAAD-that provides economic benefits and social or personal services, and promotes the coordination of all the public powers. Through this basic Law', the State guarantees to the citizens a level of minimum, common and equal protection, to which it bas the inberent responsibility (the other side of inherent power theory) to contribute with the necessary financial resources so that so equal protection is real and effective.

KEY WORDS:

Dependancy. Social services. Social security. Inberent powers. Inberent responsibilities. Constitutional competences. Responsibility of the State. Autonomous communities.

El día 15 de diciembre el Boletín Oficial del Estado publicó la Ley 39/2006, de 14 de diciembre, de Promoción de la Autonomía Personal y Atención a las personas en situación de dependencia; como anécdota podemos observar que el enunciado de la Ley se publicó en el Boletín Oficial del Estado con las mayúsculas que aquí se reproducen exactamente, que -al parecer- no reflejan ningún criterio gramatical, sino más bien la travesura de quien desearía que se popularizase el anagrama de Ley PAPA para designarla, en lugar de otras siglas más descriptivas como Ley PAAD.

A pesar de que será muy difícil evitar el reduccionismo de referirse únicamente a la dependencia, por la comodidad de la brevedad, y porque ésta es la contingencia básica que se tutela, hay que recordar que en numerosas comparecencias en distintas comisiones que en el Congreso de los Diputados abordaron el tema de la dependencia (Comisión para las políticas integrales de la discapacidad; Comisión de seguimiento y evaluación acuerdos Pacto de Toledo; Comisión de Trabajo y Asuntos Sociales) los representantes de los sectores afectados solicitaron reiteradamente una visión positiva de las acciones a emprender; de aquí que en toda la Ley se mencione la promoción de la autonomía personal antes que a la atención a la dependencia; si bien sólo en el tramo final del debate parlamentario se modificó el nombre del Sistema para la Autonomía y Atención a la Dependencia, que en todos los documentos anteriores se le había denominado únicamente Sistema Nacional de Dependencia.

La Ley PAAD ha sido aprobada en el Congreso con un amplio respaldo parlamentario, que no es sino el colofón de la unanimidad con la que todos los grupos políticos y sociales habían reclamado una mejora de la protección social a lás perso- 
nas que precisan de ayudas importantes para las actividades de su vida diaria. Dejando al margen alguna abstención a concretos artículos por cuestiones que nada tenían a ver con la protección social, la única oposición a la promulgación de la Ley la protagonizaron algunos partidos vascos y catalanes por entender que esta ley no respetaba la delimitación competencial entre la actividad del Estado y la de las Comunidades Autónomas.

Aunque a veces puede volverse tedioso, el debate competencial no puede rehuirse; es simplemente un debate más acerca de cómo podrían hacerse las cosas de la manera que cada uno cree mejor, y si a veces puede parecer complejo, es porque a menudo engloba múltiples alternativas, ya que la determinación del ámbito competencial es el resultado final de las opciones adoptadas por el legislador acerca de qué se protege, y de cómo se protege la situación que se desea tutelar.

Una de las primeras características que puede apreciarse al analizar la nueva ley PAAD es que es un texto que desde el primer al último artículo alude a una pluralidad de poderes públicos que deben concurrir y coordinarse para aplicar sus preceptos; es una ley pensada desde el reparto competencial y no simplemente respetando la existencia de otras competencias concurrentes o colindantes. No se limita a utilizar la manida expresión "sin perjuicio de las competencias que pudieran corresponder a las Comunidades Autónomas" u otra similar ${ }^{1}$, que si bien no puede afirmarse que encubra un exceso competencial, sí que deja al intérprete la tarea de averiguar cual es el papel que se reserva a los otros entes distintos a la Administración General del Estado, o cuando menos deja al margen el encaje y coordinación de las diversas actuaciones sobre una misma materia.

La Ley constituye un nuevo mecanismo tutelar dentro del campo de la protección social, al que denomina Sistema para la Autonomía y Atención a la Dependencia (en adelante: SAAD). Y parece más acertado utilizar la expresión "constituye" que no la más usual de instaura, dado que no simplemente es novedoso sino que su regulación se realiza con expresa voluntad de autonomía respecto a los existentes Sistemas

1. Tal expresión aparece centenares de veces en cualquier base de datos de legislación. Por indicar ejentplos recientes, podemos hallarla en la Ley 40/2006, de 14 de diciembre, en materia de emigración: "Los objetivos y finalidades de esta Ley se entienden, en todo caso, sin perjuicio de las competencias que ostenten las Comunidades Autónomas ni de sus leyes"; en la Ley 29/2006, de 26 de julio en materia de farmacia: "...podrá autorizarse, excepcionalmente, la creación de botiquines en las condiciones que reglamentariamente se determinen con carácter básico, sin perjuicio de las competencias que tengan atribuidas las Comunidades Autónomas en esta materia": o la Ley Orgánica 2/2006, de 3 de mayo, en materia de enseñanza: "El Ministerio de Educación y Ciencia, sin perjuicio de las competencias de las Comunidades Autónomas, podrá establecer, por sí mismo o en colaboración con otras entidades, premios y concursos de carácter estatal destinados a alumnos, profesores o centros escolares". 
de Salud, y de Seguridad Social; autonomía que se manifiesta tanto en el aspecto conceptual como en el organizativo.

Siguiendo -por fin!- una correcta técnica legislativa; el texto legal contiene en su artículo 2 las definiciones de los conceptos que se utilizan en la norma; entre ellas se hallan las que definen la contingencia que se va a proteger: la dependencia, y la autonomía que se quiere promover. Tales definiciones se formulan sin referencia alguna a otras normas españolas o internacionales; esto podría explicarse por el hecho de que se apartan de los conceptos o estándares ya formulados, por ejemplo, cuando se dice que se tutelará sólo la incapacidad de desarrollar con autonomía las actividades básicas de la vida diaria -esto es: $\sin$ las llamadas actividades instrumentales ${ }^{2}$, que sí quedarían incluidas en la definición de dependencia que utiliza el Consejo de Europa-; o también podría explicarse esta autonomía de conceptos por el hecho de utilizar conceptos novedosos como el de Tercer Sector, que no figura ni en el diccionario de la Real Academia Española, ni en la base de datos ILOTERM de la Organización Internacional del Trabajo. Más adelante podemos ver cómo al establecer las líneas maestras del baremo por el que deberá valorarse el grado de dependencia de los sujetos interesados en beneficiarse de los cuidados previstos en la ley, la norma indica que el baremo "tendrá entre sus referentes la Clasificación Internacional...", indicando una vez más la voluntad de no trasladar integramente tal criterio internacional ya establecido como estándar.

Sin embargo, el aspecto más llamativo en cuanto a la construcción autónoma del nuevo sistema protector es la cuidadosa elusión de los conceptos y preceptos del Sistema de Seguridad Social, salvo en el supuesto meramente instrumental de establecer enlaces explícitos entre situaciones de uno y otro sistema, demostrando precisamente con la necesidad de establecer estos enlaces que se trata de dos conjuntos que tienden a la autointegración, como, por ejemplo, la convalidación como afectados de dependencia a quienes tuvieran reconocida una pensión de Gran Invalidez. Este cuidadoso distanciamiento respecto al Sistema de Seguridad Social incluye la Exposición de Motivos de la ley en donde, a pesar de que sí reconoce que hasta el momento actual "el sistema de Seguridad Social ha venido asumiendo algunos elementos de atención a las situaciones de dependencia: gran invalidez, complementos de ayuda a tercera persona en la pensión no contributiva de invalidez y de la prestación familiar por hijo a cargo con discapacidad, asimismo, las prestaciones de servicios sociales en materia de reeducación y rehabilitación de personas con discapacidad y de asistencia a las personas mayores" se evita cualquier referencia al artículo 41 de la Constitución, mientras que sí se alude a los artículos 49 y 50.

2. Por ejemplo, comer seria una actividad básica diaria, y cocinar o comprar serian actividades instrumentales, para las cuales puede depender un sujeto de la ayuda de terceras personas. 
Que esta omisión es consciente lo demuestra el hecho de que en la memoria preparada por el Gobierno acompañando al anteproyecto que se remitió al Consejo Económico y Social y al Consejo de Estado, en el apartado 5.2 titulado "legitimación constitucional para la aprobación de una ley estatal" sí que se mencionaba el artículo 41 de la Constitución, en estos términos: "De forma más concreta, el artículo 9.2 establece que "corresponde a los poderes públicos promover las condiciones para que la libertad y la igualdad del individuo y de los grupos en que se integran sean reales y efectivas; remover los obstáculos que impidan o dificulten su plenitud y facilitar la participación de todos los ciudadanos en la vida política, económica, cultural y social". Asimismo, el artículo 41 alude a que "los poderes públicos mantendrán un régimen público de Seguridad Social para todos los ciudadanos, que garantice la asistencia y prestaciones sociales suficientes ante situaciones de necesidad...". El artículo 49, a su vez, obliga a los poderes públicos a realizar una política de integración social de los ciudadanos con discapacidad "para el disfrute de los derechos que éste título otorga a todos los ciudadanos". Y el artículo 50, en fin, insta a los poderes públicos a garantizar "mediante pensiones adecuadas y periódicamente actualizadas, la suficiencia económica a los ciudadanos durante la tercera edad. Asimismo. y con independencia de las obligaciones familiares. promoverán su bienestar mediante un sistema de servicios sociales que atenderán sus problemas específicos de salud, vivienda, cultura y ocio".

La reflexión acerca del encaje en la Seguridad Social de la protección a las situaciones de dependencia ha acompañado el largo proceso de elaboración de la norma. Ya en 1999 el Defensor del Pueblo recomendaba al Ministerio de Trabajo y Asuntos Sociales “... la implantación de las medidas necesarias para dar a la situación de dependencia una regulación coherente, eficaz y eficiente en el marco del sistema de Seguridad Social. (Defensor del Pueblo, 1999: 82). El dictamen del CES, al criticar la "la parquedad del fundamento constitucional en el que se ampara el Anteproyecto" se remitía a las materias que en las primeras páginas de su informe señalaban un elenco de materias que resultaban afectadas por el texto legal, entre ellas la Seguridad Social aludida en el art. 149.1.17 de la Constitución ${ }^{3}$; y en el dictamen del Conse-

3. El párrafo al que se alude dice así: "Afecra, asimismo. a una serie de materias enunciadas en diversos números del artículo 149, sobre las que el Estado tiene competencia exclusiva. A este respecto. cabe referirse sin ánimo de exhaustividad, a las cuestiones relacionadas con las bases y coordinación general de la sanidad (artículo 149.16), la legislación básica de Seguridad Social, sin perjuicio de la ejecución de sus servicios por las Comunidades Autónomas (artículo 149.17), las bases del régimen jurídico de las Administraciones públicas y del régimen estatutario de sus funcionarios que, en todo caso, garantizarán a los administrados un tratamiento común ante ellas (artículo 149.18), la obtención, expedición y homologación de títulos académicos y profesionales (artículo 149.30), o la estadistica para fines estatales (artículo 149.31)." 
jo de Estado sobre el texto del anteproyecto se dejaba constancia de que "En alguno de los informes recabados en la tramitación del expediente se alude a la preferencia manifestada en distintos ámbitos por una norma básica estatal inserta en el ámbito de protección de la Seguridad Social" .

La dependencia como contingencia no hay duda de que podría ser una de las tuteladas por la Seguridad Social, especialmente en nuestro país, desde que bajo la inspiración del concepto contenido en el art. 41 de la Constitución, no se considera ya que la Seguridad Social deba ser únicamente de base profesional, sino que comprende asimismo la tutela de riesgos sociales universales. Por ello, la doctrina ha reflexionado sobre la necesidad de que se definiera su tutela como una contingencia específica (MERCADER, 2006); y, a mayor abundamiento, puede señalarse que la Carta de Derechos Fundamentales de la Unión Europea, aprobada en el 2005, como refundición de los derechos fundamentales que se aplican en toda la Unión ${ }^{5}$ coloca en el artículo 34 a la dependencia junto a las contingencias de maternidad, enfermedad, accidentes laborales, vejez, y la pérdida de empleo.

Ciertamente la contingencia de la protección de la autonomía personal y la atención a la dependencia no es una de las clásicas contingencias que configuran el estándar comúnmente aceptado como propio de la Seguridad Social a partir del Convenio 102 de la OIT; sin embargo, en muchos estudios y especialmente en el conocido Libro Blanco sobre la "Atención a las personas en situación de dependencia en España" que el Ministerio de Trabajo encargó ${ }^{6}$, queda reflejado que en la mayoría de países europeos existen mecanismos de tutela de esta situación de necesidad, y en nuestro país existe una larga tradición de protección social de estas situaciones que se han tutelado básicamente por las administraciones locales y autonómicas.

La protección existente era, por consiguiente, muy desigual. Dependía, en primer lugar, del municipio o Comunidad Autónoma de residencia; y, en segundo lugar, de las disponibilidades presupuestarias que podían variar de un año a otro, o incluso dentro del mismo ejercicio, ya que lo característico de nuestros mecanismos de Asistencia Social y Servicios Sociales es que se prestan, no discrecionalmente, pero sí de forma condicionada a las disponibilidades existentes; los créditos presupuestarios de

4. Tramitado como Expediente 336/2007 fue aprobado el día 23 de marzo de 2006. El Boletin Oficial del Estado mantiene una base de datos con todos los dictámenes emitidos por este organismo.

5. Esta descripción de la Carta es la que se dio tras la reunión de Jefes de estado en Colonia (Alemania) en junio de 1999. Para una descripción sintética de la protección de la dependencia en Europa, véase López López, Julia (2004).

6. Dada su considerable extensión es recomendable su consulta en alguna de las múltuples páginas web que lo reproducen, por ejemplo, en: http://www.tt.mtas.es/periodico/serviciossociales/200501/ libro_blanco.pdf. 
los que se nutren no son ampliables para atender a una demanda que pueda exceder a las previsiones, y no son raras las situaciones en las que se han denegado prestaciones por agotamiento del crédito presupuestario aprobado, o se ha deferido su pago efectivo al ejercicio posterior.

Era perfectamente lógico que, en una situación en la que confluían una buena situación financiera de nuestro sistema de Seguridad Social y unas perspectivas de incremento de las situaciones de necesidad debidas al progresivo envejecimiento de la población, se plantease la incorporación de la dependencia como una nueva contingencia a tutelar. La situación guardaría una relativa semejanza con la que se produjo en 1990 cuando la Seguridad Social incorporó mediente las pensiones no contributivas por vejez e invalidez a quienes hasta el momento venían siendo tutelados por mecanismos de asistencia social cuya gestión ya había sido transferida o se hallaba camino de serlo a cada una de las Comunidades Autónomas.

Más aún: esta posibilidad de expansión de la tutela de la Seguridad Social se torna en obligación a la luz de la vocación expansiva de la Seguridad Social que dimana de los principios de generalidad objetiva y universalidad subjetiva que contiene el artículo 41 de la Constitución. Si se constata una situación de necesidad que progresivamente afecta a un mayor número de personas (la pérdida de autonomía personal con el advenimiento a una situación de dependencia de terceros para los actos vitales) y a la vez se aprecia que nuestro sistema de Seguridad Social tiene una razonable salud financiera ${ }^{7}$, el mandato constitucional de que el régimen público de Seguridad Social debe proveer de prestaciones suficientes ante situaciones de necesidad obligaba a los poderes públicos a estudiar y proponer la tutela de tal contingencia. Asimismo, si se constata que un número importante de ciudadanos se hallan afectados por una contingencia de pérdida de autonomía personal que no deriva necesariamente de una actividad profesional anterior, ni tan sólo se manifiesta en la mayoría de los casos en las edades de posible actividad laboral, el sistema de protección social deberá adecuar su ámbito protector para dar cobertura universal a tales necesidades.

Pero la Seguridad Social no es únicamente un ámbito conceptual, es también un ámbito financiero, organizativo y de gestión; y la ubicación de determinados mecanismos de protección social fuera del ámbito jurídico-organizativo denominado espe-

7. Según los informes presentados al Congreso, a 10 de marzo de 2006 el importe acumulado en el Fondo de reserva de la Seguridad Social era de 31.253.259.631,95 euros. Más detalles en: DS. Congreso de los Diputados Núm. 532 de 14/03/2006; comparecencia, a petición del Gobierno, del Secretario de Estado para la Seguridad Social. No es mucho, sólo algo más de seis meses del importe de las pensiones; pero no es poco, es más del 3\% del PIB de España; y para comparar debemos tener en cuenta que el coste del nuevo SAAD se espera que represente poco más del $1 \%$ del PIB. 
cíficamente en nuestro país "Seguridad Social" ha dado lugar a la coexistencia de dos significados paralelos de la expresión Seguridad Social: un concepto material amplio y un concepto estructural más estricto. Puede, por ello, plantearse razonablemente la duda de hasta qué punto es legítimo calificar de Seguridad Social a mecanismos de protección social que se hallen segregados del ámbito normativo delimitado por la Ley General de Seguridad Social; esta duda que ahora podríamos formular en materia de la protección a la dependencia se ha expresado anteriormente, y en razón de sus especificidades de gestión, financiación y régimen jurídico, en relación al sistema de pensiones para los funcionarios del Estado denominado Clases Pasivas, en relación a la protección sanitaria universal, e incluso late en algunos reproches de privatización que se formulan a las MATEPSS que en amplia medida gestionan la protección de las consecuencias de los accidentes de trabajo.

En el ámbito internacional se ha reflexionado bastante en los últimos años sobre las posibilidades y mecanismos para favorecer la extensión y universalización de la cobertura de la Seguridad Social, y en los diversos estudios publicados se insiste que la OIT mantiene un criterio extremadamente flexible para considerar que un sistema de protección cumple con los estándares mínimos de Seguridad Social. En el Convenio 102 de la OIT -y los posteriores convenios, los llamados de tercera generación ${ }^{8}$; tanto da que la protección de las contingencias se realice en un sistema específico, como en varios regímenes paralelos o conexos, como los de sanidad o asistencia social, incluso si parte de dichos mecanismos tutelares son privados o facultativos; lo que importa es la proporción de ciudadanos efectivamente protegidos, y no las fórmulas financieras, organizativas o legales; asimismo no se ve ningún obstáculo en que un sistema de seguridad social incluya contingencias como ayudas alimentarias, formación e inserción, prevención sanitaria o ayudas a personas ancianas. Evidentemente, el que una prestación sea universal, y que sus prestaciones se calculen prescindiendo de todo el posible historial laboral anterior tampoco es extraño al concepto de seguridad social, ya que en el propio Convenio 102 y posteriores se contemplan tres modelos típicos de cálculo de las prestaciones: un porcentaje de los ingresos anteriores, una prestación uniforme, o un baremo según la necesidad acreditada (HuMBlet 2002: 11).

Una objeción distinta frente a considerar la protección de la dependencia como propia de la Seguridad Social sería el hecho de que la Ley prevé primordialmente prestaciones en especie que pueden clasificarse como servicios sociales, esto es:

8. Hace referencia a las características estructurales comunes de los convenios a partir de 1952. La primera generación serian los convenios adoptados en el periodo 1919-1944 basados en los seguros sociales como método; la segunda generación, en el periodo 1944-1952 con un concepto global y amplio de Seguridad Social para constituir un sistema único Véase: Javillier, J:C. (2002; 455 y ss). 
servicios técnicos prestados de forma continuada y susceptibles de uso colectivo? . A partir de esta constatación podría argumentarse que los Servicios Sociales son una materia distinta a la Seguridad Social, como lo muestra el que los Servicios Sociales se hallan transferidos a las Comunidades Autónomas, mientras que la Seguridad Social es una materia normada y gestionada por el Estado; en este sentido dos consejeros del CES formularon un voto particular al dictamen de la Ley que comentamos en el que se decía "...la Ley le otorga al ejecutivo español todo lo concerniente a la toma de decisiones sobre el nivel de cobertura mínimo garantizado, invadiendo así las competencias de las instituciones de Galiza, Navarra y de la CA del País Vasco en los servicios sociales, que son exclusivas en esta materia."

Tal objeción puede contradecirse en sus dos proposiciones, ya que ni es claro que el SAAD sea un sistema de servicios sociales, ni tampoco que los servicios sociales sean una materia exclusiva de las CCAA, diferenciada de las materias de competencia estatal. Aun cuando es cierto que en la ley se manifiesta en algún caso una preferencia por las prestaciones en especie, por cuanto las prestaciones económicas se hacen subsidiarias de una posible prestación técnica, y en todo caso se afectan al pago de servicios, lo cierto es que existen tres categorías de prestaciones: prestaciones económicas, servicios sociales de uso potencialmente colectivo, y servicios personales; es decir, individualizados; por ello la Ley expresa claramente (art. 14.1) que "las prestaciones de atención a la dependencia podrán tener la naturaleza de servicios y de prestaciones económicas". Por otra parte. hasta el momento actual las Comunidades Autónomas habían asumido las competencias en materia de Servicios Sociales pero no como algo ajeno a la Seguridad Social sino -al menos en partevinculadas a ella, ya que el ejercicio de tales competencias se inició mediante el traspaso de los Servicios Sociales que se hallaban afectos a ella, y posteriormente se ha asumido el conjunto de la materia mediante una mezcla de las posibilidades que brindaban los artículos 149.1.17 (ejecución de los servicios), 148.1.20 (asistencia social) y 149.3 (lo no reservado expresamente al Estado). Se da la paradoja de que en el preámbulo de las actuales leyes autonómicas de servicios sociales, que regulan una competencia que absolutamente todas las CCAA ejercen, se expresa de forma desigual el fundamento constitucional que ampara tal competencia.

9. Esta es una de las definiciones académicas al uso, si bien debe observarse que todas las leyes autonómicas de servicios sociales han rehuido efectuar una definición sintética de los mismos, debiendo deducirse del conjunto de su articulado. Probablemente esta indefinición se corrija a partir de la nueva ola de textos estatutarios que ahora de forma más generalizada mencionan los Servicios Sociales como competencia asumida con exclusividad (con dudoso acierto aunque copiado miméticamente de los primeros textos revisados). Tampoco la Ley General de Seguridad Social (art. 53) define un concepto de Servicios Sociales. 
En los años anteriores mucho se ha escrito sobre la posible relación o no entre la Asistencia Social y la Seguridad Social ${ }^{10}$ y, a tenor de las primeras escaramuzas, va a reproducirse el debate con la relación entre los Servicios Sociales y la Seguridad Social. A este respecto hay que recordar que los criterios internacionales no son contrarios a una concepción unitaria de Seguridad Social y Servicios Sociales, como puede verse en este fragmento aprobado en la $89^{a}$ reunión de la Conferencia Internacional del Trabajo (OIT, 2001): "3. La seguridad social, administrada correctamente, aumenta la productividad al proporcionar asistencia médica, seguridad de ingresos y servicios sociales.". Por otra parte, desde hace muchos años se ha defendido doctrinalmente y con rigor (Alarcón, 1986: 16-22) una visión integradora de los Servicios Sociales dentro de la Seguridad Social, sin perjuicio de un desarrollo normativo y ejecutivo autonómico sólo limitado por la legislación básica y los conceptos transversales que la Constitución reserva al Estado.

No puede ignorarse que existen tendencias que tienden a reducir progresivamente el ámbito de lo que debe entenderse por Seguridad Social mediante distintas vías. La primera de ellas es considerar como extraño a la Seguridad Social las prestaciones que se universalizan, y así sucedió con la asistencia sanitaria que vio cuestionada su pertenencia al ámbito de la Seguridad Social a partir del momento en que se dictaron las disposiciones que extendieron su ámbito de cobertura a toda la población; este criterio abocaría a la Seguridad Social a "morir de éxito": en la medida en que se alcanzase en un ámbito prestacional el objetivo -constitucionalmente impuesto- de universalidad subjetiva vería mutilarse tal ámbito para ubicarlo en una zona más amplia de política social. Para justificar esta segregación no vale argumentar una teórica contraposición entre derechos de ciudadanía y derechos sociales o de protección social, puesto que el artículo 41 de la Constitución quiere una Seguridad Social para todos los ciudadanos; esta expresión de "ciudadanos", significa -como se ha escrito repetidamente- que es más amplio que los viejos criterios profesionales delimitadores del ámbito subjetivo, pero es también un criterio mucho más concreto que las amorfas expresiones de "todos" o "todas las personas" que se utilizan en otros párrafos constitucionales: al referirse a los beneficiarios de la Seguridad Social como ciudadanos, significa que se integran los derechos y las obligaciones de la protección social en el universo de derechos y obligaciones de la ciudadanía.

Naturalmente, una Seguridad Social para todos los ciudadanos se gestiona por quienes han sido elegidos por todos los ciudadanos tras someter a su voto un determinado programa, y no únicamente -aunque sí pueden participar- por agentes sociäles que emanan de un sector profesional o societario; es un sistema que se financia

10. Mi posición, sintéticamente, es considerar la Asistencia Social como un área secante con la Seguridad Social no básica, y la competencia autonómica vendría amparada por un doble título competencial, puede verse con más detalle en Fargas (2002: 349-383), 
mediante una pluralidad de impuestos, especialmente afectados -como las cotizaciones- o no, y en la que los ciudadanos pueden decir que tienen derecho a las prestaciones porque -utilizando la peliculera frase- pagan sus impuestos; y un sistema para todos los ciudadanos debe ser también un sistema permeable y adaptable a las situaciones de necesidad que se crean o se agravan para el conjunto de la ciudadanía.

Otro tipo de argumentación que tiende a limitar el crecimiento de la Seguridad Social es considerar extrañas a la misma las prestaciones que no sean monetarias. No se niega la posibilidad de prestaciones en especie, la LGSS las prevé. pero se consideran como una excepción, la excepción que confirma la regla: porque lo que se considera propio de la Seguridad Social es repartir dinero. Para ello se parte de una constatación irrefutable: la vigencia -cuantitativamente abrumadora- del histórico criterio de que la Seguridad Social sirve para solventar las situaciones ocasionadas por unos riesgos sociales que ocasionan un exceso de gastos o un defecto de ingresos. Sin embargo, mantener como exclusivamente propia esta técnica tradicional entre las que pueden utilizarse por la Seguridad Social omite reconocer que nos hallamos en una sociedad que evoluciona rápidamente -por ejemplo envejeciendo-, en la que debido a tal evolución aparecen nuevas demandas -por ejemplo mayor asistencia sanitaria, más atención a la dependencia-, y en la que se han transformado las estructuras sociales y familiares a cargo de las cuales corría la atención de enfermos y dependientes; y perfectamente podría pensarse más bien que esta evolución social debe empujarnos a propugnar cambios en la concepción de lo que debe ser la Seguridad Social.

Justamente las necesidades cuya demanda cle tutela más aumenta: la sanidad y la atención a los dependientes, sólo pueden ser satisfechas con criterios de máxima suficiencia mediante prestaciones públicas y universales en especie. De la misma forma que, a pesar de las cíclicas saturaciones que sufren, nadie piensa actualmente que los servicios públicos de urgencias hospitalarias podrían ser suplidos eficazmente mediante el reparto de una prestación económica, las nuevas demandas de atención a la dependencia sólo podrán ser satisfechas -si pensamos en términos de suficiencia universal- mediante prestaciones públicas en especie, y mediante servicios sociales. Ello no quiere decir que siempre deba ser así, ya que es lógico que haya un número respetable de situaciones suficientemente nuevas, o minoritarias, o especiales, para las que no puedan arbitrarse servicios instituidos con anterioridad y deba recurrirse a pagar en metálico la solución ad-boc personalizada, cuando no improvisada. En resumen: las demandas emergentes de protección social requieren reforzar las prestaciones en especie de la Seguridad Social, no su extrañamiento allende muros del sistema.

Las prestaciones que debe proporcionar la promoción de la autonomía personal y tutela a la dependencia no pueden ser básicamente económicas, también debido al hecho de que debe tutelarse a dos grandes tipos de personas afectadas: $a$ ) los aquejados por la limitación o pérdida de su capacidad de realizar personalmente las 
actividades básicas o instrumentales de la vida diaria; y b) las personas que conviven más estrechamente con quienes presentan tales limitaciones personales, y que en primera instancia han sido sus cuidadores, sacrificando su desarrollo personal, salud, posibilidades profesionales, y participación social. Limitarse a incrementar la renta de las personas del primer grupo, en la mayoría de los casos no serviría para tutelar adecuadamente al segundo grupo, el de las personas hasta ahora cuidadoras. $\mathrm{Y}$ de ello no puede deducirse que los servicios no sean propios de la Seguridad Social, sino más bien lo contrario: parece que la Seguridad Social debería incrementar su oferta de servicios, de prestaciones en especie.

Una tercera línea de objeciones tiende a contener la generalidad objetiva que debería conseguir nuestro sistema de Seguridad Social según se deduce del artículo 41 $\mathrm{CE}$, oponiendo la distribución competencial que permite a las Comunidades Autónomas asumir las competencias en materia de sanidad, asistencia social y servicios sociales, y para ello se simplifica que la Constitución reserva la Seguridad Social al Estado y lo que no es Seguridad Social es lo que corresponde a los poderes públicos autonómicos. Frente a ello hay que repetir que el 149.1.17 reserva al Estado, además del régimen económico, únicamente la legislación, y no toda la legislación, sino -sólo y toda- la que pueda considerarse básica de la Seguridad Social; de la dificultad doctrinal o política de llenar de contenido la posible legislación no básica no puede deducirse que el concepto de Seguridad Social debe identificarse con aquello que el Estado realice; ni tampoco de la dificultad doctrinal o política para identificar y traspasar las actividades de ejecución que pueden y deben realizarse por las Comunidades Autónomas.

Por el contrario, y como ya se ha dicho, es posible sostener una visión en la que la Seguridad Social, la Asistencia Social, y los Servicios Sociales no son materias fronterizas, sino secantes; y que el pluralismo de poderes que deben concurrir a la construcción del estado social exige considerar que el sistema de Seguridad Social aludido en el art. $41 \mathrm{CE}$ es un sistema normativo y de gestión amplio y plural, del que deben responsabilizarse y al que deben contribuir toda la red de poderes públicos, que puede comprender a varios subsistemas, y, por consiguiente, el Estado puede ampararse en el artículo 149.1.17 para crear el SAAD. Y ello aunque en el texto de la Ley no se haya mencionado tal fundamento, ya que, como oportunamente recuerda el Consejo de Estado en su dictamen, según el Tribunal Constitucional [STC 233/1999 y STC 164/2001] nos hallamos frente a una "inexistente obligación del legislador de invocar expresamente el título competencial que ampara sus leyes".

En la Ley se menciona únicamente el artículo 149.1.1 de la Constitución como amparo de la actuación estatal. Este artículo, que encomienda al Estado la garantía de las condiciones básicas que garanticen la igualdad de todos los españoles en el ejercicio de los derechos y obligaciones, ha sido de los más citados por el abogado del Estado en los recursos ante el Tribunal Constitucional contra normas autonómiêas; y 
normalmente se ha visto repetir una y otra vez que es un artículo que por si sólo no basta para atribuir competencias al Estado, sino que debe servir más bien para modular el ejercicio de las competencias basadas en otros preceptos; y especificamente responsabiliza al Estado de que en el ejercicio de sus potestades de legislación básica tenga como guía el garantizar esta garantía de condiciones de igualdad. El Tribunal Constitucional precisó que la garantía "se ha de materializar 'a través de', no 'a pesar de' los sistemas de reparto de competencias articulados en la Constitución, de manera que la promoción de la igualdad sustancial y la acción estatal destinada al efecto debe desplegarse teniendo en cuenta las peculiaridades de un sistema de autonomías territoriales"11. Por este motivo hemos puesto énfasis en argumentar que el contenido normado en la ley que analizamos puede entenderse comprendido en la materia Seguridad Social respecto a la cual el Estado, en virtud del art. 149.1.17 ostenta las potestades de legislación básica, y las Comunidades Autónomas pueden ejercer la normación no básica y las potestades ejecutiva y de autoorganización.

Debemos recordar que la responsabilidad de promover la igualdad real y efectiva entre todos los individuos y grupos es de todos los poderes públicos y no sólo del Estado [art. 9.2 CE], al Estado le compete el objetivo de garantizar unas condiciones básicas comunes [art. 149.1.1 CE]; de forma paralela compete a todos los poderes públicos garantizar asistencia y prestaciones sociales suficientes [art. $41 \mathrm{CE}$ ], y al Estado en particular dictar la legislación básica de la Seguridad Social [149.1.17 CE].

El procedimiento utilizado en la Ley ha sido, en primer lugar, el de limitar el alcance de la ley a los aspectos que puedan considerarse básicos. esto es, sin agotar la regulación, y dejando un considerable margen de diferenciación autonómica. Esta autolimitación estatal se hace patente no sólo en el hecho de que la redacción del texto legal no sea minucioso en grado tal que no permitiera desarrollo normativo alguno por parte de la legislación autonómica, sino también de forma destacable por las amplias facultades [art. 8] que se atribuyen al Consejo territorial del SAAD formado mayoritariamente por representantes de las Comunidades Autónomas, y por el traspaso ex-lege de todos los servicios y prestaciones económicas previstas en la ley a la red de servicios de las respectivas Comunidades Autónomas [art. 16] que podrán ejercer todas las facultades de ejecución, autoorganización y -limitadamente-las de desarrollo normativo. Por consiguiente, los reproches que en sede parlamentaria ${ }^{12}$ se

11. STC 146/1986, de 25 de noviembre; STC 152/1988, de 20 de julio, entre otras.

12. Véanse las tropecientas preguntas formuladas al Ministerio de Trabajo en la primera mitad del 2006 que pueden consultarse en el Diario de Sesiones del Congreso, Comisión de Trabajo y Asuntos Sociales; por ejemplo en los de los días 26 y 27 de abril, 10 de mayo y 7 de julio. Más sorprendentes -por contradictorios- resultan similares reproches en el voto particular de los representantes sindicales del CIG y ELA en el dictamen del Consejo Económico y Social, inmediatamente antes de quejarse porque el texto -dicen-intenta imponer una legislación estatal uniforme al conjunto del Estado español. 
realizaron al Gobierno de que en la ley quedaban muchos detalles por concretar acerca de las condiciones, plazos e instancias para hacer efectivos cada uno de los derechos que en la ley se reconocen tienen, en primer lugar, un carácter antiautonómico, porque muchas de las concreciones competen a las Comunidades Autónomas y si figurasen el la ley básica cercenarian sus posibilidades normativas; y en segundo lugar, son extemporáneas, como lo serían las exigencias de concreción de los horarios de circulación de trenes en la discusión del plan director ferroviario.

Además de la autolimitación reseñada en la concreción del contenido material de los derechos hasta tanto no se constituyan los mecanismos de cooperación y participación autonómica y local, la articulación de los distintos poderes públicos se ha plasmado en el diseño de tres niveles de intervención distintos en cuanto a la relación entre el Estado y las Comunidades Autónomas, tres niveles que se describen sumariamente en el artículo 7 de la Ley y se desarrollan en los artículos posteriores de la Ley: a) un primer nivel mínimo, uniforme y financiado exclusivamente por el Estado: este nivel garantiza primordialmente la igualdad básica a la que alude el artículo 149.1.1 CE. b) un segundo nivel. de financiación compartida entre el Estado y las Comunidades Autónomas, que completará el nivel mínimo anterior: es el nivel que introduce diversidad, pero basada en criterios objetivos comunes que se designan en el art. 32.3 (población, dispersión, insularidad, emigrantes, etc.); y c) un tercer nivel, de financiación autonómica, potestativo y complementario de los anteriores: es el nivel en el que cada CCAA puede introducir sus criterios y alternativas, y en el que la diversidad ya puede obedecer criterios dispares de prioridad y oportunidad.

Puede constatarse que cada uno de estos tres niveles de actuación va íntimamente relacionado con tres fórmulas de financiación distintas; ante este compromiso financiero debemos plantearnos si el Estado ha actuado correctamente al asumir la financiación exclusiva del primer nivel y la financiación compartida del segundo nivel complementada con compromiso transitorio hasta el año 2015 para asegurar la implantación progresiva del SAAD. A este respecto debemos recordar la doctrina tradicional del Tribunal Constitucional de que los mecanismos financieros deben seguir a la determinación competencial y no al revés, de forma que la capacidad de gasto de una Administración Pública no es un título competencial autónomo ni determina su competencia en relación a una materia ${ }^{13}$; es evidente que la tesis contraria haría depender la distribución de competencias de la capacidad financiera que en un determinado momento estuviera a la disposición de un ente, y no de lo preceptuado en la Constitución.

Si el Estado ha decidido dictar una norma que regule el contenido básico de la promoción de la autonomía personal y la atención a la dependencia, y consideramos

13. Doctrina repetida en muchas sentencias (más de una docena) desde las STC $39 / 1982$, dé 30 de junio, y $179 / 1985$, de 19 de diciembrè; entre las más recientes podemos citar la $126 / 2002$, de 23 de mayọ, y la STC $48 / 2004$, de 25 de marzo. 
que -entre otros, seguramente- se halla legitimado para ello por el artículo 149.1.17 $\mathrm{CE}$, modulado por el objetivo de igualdad preceptuado en el artículo 149.1.1, se hallaría también el Estado legitimado para establecer los mecanismos financieros correspondientes que sin duda van a resultar indispensables para la efectiva implantación de las prestaciones previstas en la Ley. Sin embargo, decir simplemente que el Estado se hallaba facultado para ello es quedarse a medio camino, y para apreciarlo basta con pensar la posibilidad contraria: que el Estado hubiera normado las condiciones básicas de atención igualitaria a las situaciones de dependencia. estableciendo un estándar mínimo y uniforme a satisfacer por todos los poderes públicos, pero sin aportar financiación alguna para ello.

Esta posibilidad armonizadora de un estándar mínimo de prestaciones, aún sin financiación, sería probablemente correcta desde un punto de vista competencial, a la luz de lo que prevé el artículo $150.3 \mathrm{CE}$, aunque para dictar tal disposición armonizadora debiera haber seguido un iter legislativo especial, al requerirse una mayoría reforzada de ambas cámaras legislativas que apreciasen su necesidad. Tal posibilidad, empero, seria dudoso que estuviera de acuerdo con el mandato del artículo 9.2 que ordena que la promoción de la igualdad sea real y efectiva y no meramente nominal, y la efectividad de los derechos que en la Ley se reconocen obligan a prever los mecanismos financieros que los hagan posibles. de forma que la facultad que se prevé en el artículo 149.1.1 CE de regular las condiciones básicas cle igualdad de los españoles, al ponerse en relación con el artículo 9.2 CE hacer surgir una obligación implícita de asegurar financieramente aquello que se regula, por lo que cabe afirmar que el Estado no sólo podía, sino que debia instaurar una participación financiera paralela al grado de concreción normativa que regula en cada uno de los niveles de intervención previstos en el artículo 7 de la Ley de atención a la dependencia.

No es la financiera la única obligación implicita que podemos hallar en las potestades que la Constitución reconoce al Estado ${ }^{1+}$. Si relacionamos cada una de las características esenciales que el artículo $41 \mathrm{CE}$ establece para nuestro sistema de Seguridad Social, y los relacionamos con otros preceptos constitucionales, como el

14. Existe un significado de "obligación implicita" usado habitualmente en el ámbito contable: por ejemplo, en la Norma Internacional Contable $n^{\circ} 19$ sobre pagos a empleados, se refiere a obligaciones comprometidas por una obligación basada en las prácticas habituales de la misma, o por el deseo de actuar de forma equitativa. No es éste el significado en el que aqui se usa, si no más bien como la otra cara de la teoría jurídica de los "poderes implícitos" (inherent poucer) por el que se asignan las potestades necesarias para la realización de competencias $u$ objetivos expresos; aplicada sin cita expresa en nuestra jurisprudencia constitucional aunque citada en las STC 66/1985 y 108/1986, y criticada en un voto en la STC 56/1986. El concepto es usual en Derecho internacional y en documentos de la OIT, por ejemplo, los emanados del Grupo de Trabajo tripartito de alto nivel sobre las normas relativas al trabajo marítimo; también se ha utilizado en las STJCE tras la sentencia Fédéchar de 1956. El artículo 308 CE relativo a los poderes implícitos se emplea como fundamento jurídico cuando una acción de la Comunidad resulta necesaria para lograr, en el funcionamiento del mercado común, uno de los objetivos de la Comunidad. 
10.1; el 50, o el 138.1 CE, pero muy especialmente con los citados artículos 9.2 y 149.1.1 CE surge un bloque de obligaciones implícitas para llenar de contenido la Seguridad Social como "función del Estado", entendida como responsabilidad última del Estado para asegurar, además del principio de igualdad ya analizado, la dignidad personal, solidaridad, suficiencia, y promoción de la participación de los sectores afectados, yespecialmente- el principio de extensión progresiva de la Seguridad Social.

Aunque podría mencionarse algún otro principio, se destacan aquí los anteriormente citados porque, además de formar parte de nuestra Constitución, integran una lista de principios que destacada doctrina (SLPIOT, 2006:167; GolDIN, 2006: 149) ha considerado como los que deberían integrar la base común para iniciar un programa de extensión de la Seguridad Social; programa cuyo análisis y crítica no corresponde hacer aquí, pero que es oportuno citar en la medida que tiene algunos puntos de contacto con la Ley que comentamos: una concepción flexible de las materias tuteladas por la Seguridad Social. y una autolimitación del contenido normado directamente, para que las instancias inferiores más cercanas a los ciudadanos sean las que apliquen una estrategia pactada y evaluada en conjunto.

Esta metodología a su vez ha tomado algunos referentes de la técnica políticonormativa europea denominada Método Abierto de Coordinación. Para apreciar tales puntos podemos fijarnos en cómo diseñan los artículos 8.2, 10. 34, 35 de la Ley las funciones del Consejo Territorial del SAAD: respeto a la autonomía de ejecución; pacto de objetivos, planes, y método de cooperación informativa; adopción de criterios comunes de actuación, y evaluación por el propio conjunto; y como colofón de todo ello una tendencia a la que en el futuro deberemos prestar atención: una especial consideración a los medios empleados más que a la medida de los resultados.

Utilizar la técnica de evaluar los medios empleados en lugar de los resultados obtenidos, puede inquietar por cuanto parece que no garantiza que una determinada política social sea eficaz; sin embargo, es la técnica más usada para evaluar las prestaciones basadas en servicios, ya que la verificación de si una actuación en el campo de la protección social es correcta y suficiente debe referirse a menudo a los métodos empleados, a la lex artis aplicada por los dispensadores de prestaciones y servicios.

Recopilando, la Ley de promoción de la autonomía personal y de atención a las personas en situación de dependencia sigue las tendencias internacionales actuales de extensión de la Seguridad Social, entendida ésta como un sistema amplio e integrador de diversos subsistemas -entre ellos el nuevo SAAD-, que proporciona prestaciones económicas y servicios, y promueve la coordinación de todos los poderes públicos. A través de esta Ley básica, el Estado, amparado en la conjunción de los artículos 149.1.17 y 149.1.1 CE, garantiza a los ciudadanos un nivel de protección mínimo, común e igual; y la tesis que aquí se defiende es que el Estado tiene la obligación implícita de aportar los recursos financieros necesarios para que tal igual protección sea real y efectiva. 


\section{BIBLIOGRAFÍA}

Alarcon Caracuel, Manuel Ramón, "La integración de los Servicios Sociales y la Asistencia Social en la Seguridad Social”, Temas Laborales, n 7, 1986, pp. 9-39.

Barcelón Cobedo, Susana y Quintero lima, M. Gema "Las situaciones de dependencia personal como nueva contingencia protegida por el Sistema de Protección Social", Revista del Ministerio de Trabajo y Asuntos Sociales, n. 60, 2006, pp. 13-33.

Defensor Del Pueblo, "Recomendación 82/1999, de 29 de noviembre, sobre la atención sociosanitaria y aspectos conexos" BOCG. Cortes Generales. VII Legislatura. Serie A. Núm. 69, p. 82.

Fargas Fernández, Josep, Análisis crítico del sistema español de pensiones no contributivas, Navarra: Aranzadi, 2002.

Goldin, ADRIAN, "Extender la cobertura de la seguridad Social: una propuesta normativa", Relaciones Laborales, $n^{\circ} 15 / 16,2006$, pp. 149-165. El mismo texto de GolDIN, con tan sólo ligeras variaciones en: "Normas internacionales y extensión de la cobertura de la Seguridad Social: una propuesta internacional" Universidad de San Andrés (AR) http://www.udesa.edu.ar/files/UAHumanidades/DT/DT37GOLDIN.PDF. La versión francesa en: Semaine Sociale Lamy, $\mathrm{n}^{\circ} 1272,2006$, pp. 83-89; versión inglesa en: Comparative Labor Law and Policy Journal, Univ. Illinois, College of Law, Vol. 27, $\mathrm{n}^{\circ} 2,2006$, pp. 257-272.

Humblet, Martine y Silva, Rosinda, Des normes pour le XXIe siècle: Sécurité Sociale, Ginebra: OIT, 2002. p. 11

JAVILLIER, J.C. (Dir) Les normes internationales du travail: une approche globale, Ginebra: OIT, 2002, pp. 455 y ss.

LÓPEZ LOPEZ, JuLIA "Dependency and equal treatment: rethinking European Union law on family policy". INTELL 7, Kyoto (IP), 2004.

Mercader Uguina, Jesús R. "Concepto y concepciones de la dependencia" en: González Ortega, Santiago, y Quintero lima, M. Gema (Coord.) Protección social de las personas dependientes, Madrid: La Ley, 2004, pp. 63-91.

Organización Internacional del Trabajo, "Informe de la Comisión de la Seguridad Social", actas de la 89 ${ }^{2}$ Conferencia Internacional, 2001, http://www.ilo.org/public/spanish/standards/relm/ilc/ ilc89/pdf/pr-16.pdf

Súpiot, Alain, "Esbozo de un acuerdo marco relativo a la extensión de la protección social" Relaciones Laborales, $n^{\circ}$ 15/16, 2006., pp. 167-189. 